\title{
KSHV reactivation in post-transplant Kaposi sarcoma
}

\section{To the editor:}

In their article on post-transplant Kaposi sarcoma, Barozzi et al. ${ }^{1}$ elegantly show that in five of the eight Kaposi sarcoma patients investigated, tumor cells infected with Kaposi sarcoma-associated herpesvirus (KSHV) display phenotypic and genotypic features of donor origin. Based on these findings, the authors advocate the use of donor-derived, KSHV-specific T cells for the treatment of post-transplant Kaposi sarcoma.

The medical relevance and clinical implications of these findings need to be evaluated in the appropriate context, however. In particular, the seroepidemiological framework underlying the occurrence of post-transplant Kaposi sarcoma in different geographic areas must be considered.

We have previously shown that in northern Italy, where the seroprevalence of KSHV among organ donors and recipients is $4 \%$ and $6 \%$ respectively (C.P. et al., unpublished data), posttransplant Kaposi sarcoma has an incidence of $0.9 \%$, and $90 \%$ of those cases occur in patients who were seropositive at the time of transplantation ${ }^{2}$. Our data are in agreement with other retrospective studies $^{3,4}$. We can thus infer that KSHV reactivation is the most important pathogenetic event ( $>80 \%$ incidence) underlying postrenal transplant Kaposi sarcoma in the western world.

In this regard, it is noteworthy that in the only Kaposi sarcoma patient studied by Barozzi et al. with unequivocal serologic evidence of reactivation, the findings argue against a donor origin of Kaposi sarcoma tumor cells. We therefore advise that baseline serologic testing for KSHV be performed in all transplant donors and recipients to distinguish between viral transmission and reactivation in case of Kaposi sarcoma development. This should allow rational use of investigational immunotherapeutic strategies based on donor-derived, as opposed to recipient-derived, KSHVspecific T cells.

\section{COMPETING INTERESTS STATEMENT}

The authors declare that they have no competing financial interests.

Carlo Parravicini ${ }^{1}$, Chiara Scalamogna ${ }^{2}$, Sara Calattini ${ }^{2}$, Francesca Poli ${ }^{3}$, Mario Scalamogna ${ }^{3}$, Massimo Galli ${ }^{2}$, Mauro Moroni ${ }^{2} \&$ Mario Corbellino ${ }^{2}$ ${ }^{1}$ Department of Pathology and ${ }^{2}$ Institute of Infectious Diseases and Tropical Medicine, Luigi Sacco Hospital, University of Milan, Milan 20157, Italy. ${ }^{3}$ Nord Italian Transplant, IRCCS Ospedale Policlinico, Milan 20122, Italy.

\section{Luppi et al. reply:}

We would like to thank Parravicini et al. for their interest in our work. In their correspondence, they argue that the majority of transplant Kaposi sarcoma cases are due to reactivation of a preexisting infection with KSHV, rather than caused by transmission of the virus or virus-infected cells from the organ donor to the recipient, as described in our paper. Parravicini et al. stress that in the case of a reactivated viral infection, our suggestion to use KSHVspecific $\mathrm{T}$ cells for adoptive therapy would obviously have to be based on recipient-derived, rather than donorderived, $\mathrm{T}$ cells.

We agree entirely with Parravicini et al. that the majority of transplant Kaposi sarcoma cases may be the consequence of a reactivated viral infection. This was, however, not the point of our paper, which described transmission of a KSHVinfected cell from donor to recipient that could subsequently lead to the development of a Kaposi sarcoma lesion. As for the frequency of donor-derived versus recipient-derived KSHV in the pathogenesis of transplant Kaposi sarcoma, we have recently reviewed the published literature on this issue ${ }^{5}$. It appears that of 50 well-documented and published cases of transplant Kaposi sarcoma, 16 (32\%) could have been caused by donor-derived virus. Therefore, donor-derived virus may be responsible for the development of transplant Kaposi sarcoma more frequently than suggested by Parravicini et al., and this figure may depend on local prevalence of KSHV. Serologic testing for KSHV before and after transplantation may indeed be helpful, as mentioned by Parravicini et al. In addition, in order to substantiate the origin of KSHV in a transplant patient with Kaposi sarcoma, peripheral blood DNA from the donor should be stored at the time of transplantation. This would allow the comparison of KSHV strains in the donor and the recipient by sequencing of the hypervariable $K 1$ gene or by restriction fragment-length polymorphism analysis ${ }^{6,7}$.

Mario Luppi ${ }^{1}$, Patrizia Barozzi ${ }^{1}$, Fabio Facchetti ${ }^{2}$, Thomas F. Schulz ${ }^{3}$ \& Giuseppe Torelli ${ }^{1}$

${ }^{1}$ Department of Oncology and Hematology, University of Modena and Reggio Emilia, Modena 41100, Italy. ${ }^{2}$ Department of Pathology, University of Brescia 25124, Brescia, Italy. ${ }^{3}$ Department of Virology, Hannover Medical School, Hannover 30625, Germany.

1. Barozzi, P. et al. Post-transplant Kaposi sarcoma originates from the seeding of donor-derived progenitors. Nat. Med. 9, 554-561 (2003).

2. Parravicini, C. et al. Risk of Kaposi's sarcoma-associated herpesvirus transmission from donor allografts among Italian posttransplant Kaposi's sarcoma patients. Blood 90, 2826-2829 (1997).

3. Farge D. et al. Human herpes virus- 8 and other risk factors for Kaposi's sarcoma in kidney transplant recipients. Groupe Coopératif de Transplantation d'lle de France (GCIF). Transplantation 67, 1236-1242 (1999).

4. Euvrard, S. et al. Skin cancers after organ transplantation. N. Engl. J. Med. 348, 1681-1691 (2003).

5. Luppi, M. et al. Human herpes virus-8 (HHV-8) associated diseases in solid organ transplantation: importance of viral transmission from the donor. Clin. Infect. Dis., in press.

6. Luppi, M., et al. Bone marrow failure associated with human herpesvirus 8 infection after transplantation. New Engl J Med 343, 1378-1385 (2000).

7. Luppi, M., et al. Molecular evidence of organ-related transmission of Kaposi sarcoma-associated herpesvirus or human herpesvirus- 8 in transplant patients. Blood 96, 3279-3281 (2000). 